\title{
Progression of Inner Ear Pathology in Ames Waltzer Mice and the Role of Protocadherin 15 in Hair Cell Development
}

\author{
Karen S. Pawlowski, ${ }^{1,2,3}$ Yayoi S. Kikkawa, ${ }^{1}$ Charles G. Wright, ${ }^{1,3}$ and Kumar N. Alagramam ${ }^{4}$ \\ ${ }^{1}$ Department of Otolaryngology-Head and Neck Surgery, University of Texas Southwestern Medical Center, Dallas, TX 75390, \\ USA \\ ${ }^{2}$ Graduate School of Biomedical Sciences, University of Texas Southwestern Medical Center, Dallas, TX 75390, USA \\ ${ }^{3}$ Callier Center, Behavior and Brain Sciences, University of Texas at Dallas, Dallas, TX 75235, USA \\ ${ }^{4}$ Department of Otolaryngology-Head and Neck Surgery, University Hospitals of Cleveland, Case Western Reserve University, \\ Cleveland, $\mathrm{OH}$ 44106, USA
}

Received: 9 February 2005; Accepted: 30 November 2005; Online publication: 12 January 2006

\begin{abstract}
The Ames waltzer (av) mouse mutant exhibits auditory and vestibular abnormalities resulting from mutation of protocadherin 15 (Pcdh15). Ames waltzer has been identified as an animal model for inner ear pathology associated with Usher syndrome type $1 \mathrm{~F}$. Studies correlating anatomical phenotype with severity of genetic defect in various $a v$ alleles are providing better understanding of the role played by Pcdh 15 in inner ear development and of sensorineural abnormalities associated with alterations in $P c d h 15$ protein structure as a result of gene mutation. In this work we present new findings on inner ear pathology in four alleles of $a v$ mice with differing mutations of Pcdh15 as well as varying alterations in inner ear morphology. Two alleles with in-frame deletion mutations $\left(P c d h 15^{a v-J}\right.$ and $\left.P c d h 15^{a v-2 J}\right)$ and two presumptive functional null alleles (Pcdh15 $5^{a v-3 J}$ and $P c d h 15^{a v-T g}$ ) were studied. Light and electron microscopic observations demonstrated that the severity of cochlear and vestibular pathology in these animals correlates positively with the extent of mutation in Pcdh15 from embryonic day 18 (E18) up to 12 months. Electron microscopic analysis of immature ears indicated early abnormalities in the arrangement of stereocilia and the inner and outer hair cell cuticular plates, stereo-
\end{abstract}

Correspondence to: Karen S. Pawlowski - Department of Otolaryngology • University of Texas Southwestern Medical Center • Dallas, TX 75390-9035, USA. Telephone: 214-648-6821; fax: 214-648-9122; email: Karen.Pawlowski@utsouthwestern.edu cilia rootlets, and the actin meshwork within the cuticular plate. In severe cases, displacement of the kinocilium and alterations in the shape of the cuticular plate was also observed. Mice harboring inframe deletion mutations showed less disorganization of stereocilia and cuticular plates in the organ of Corti than the presumptive functional null alleles at $\mathrm{P} 0-\mathrm{P} 10$. A slower progression of pathology was also seen via light microscopy in older animals with inframe deletions, compared to the presumptive functional null mutations. In summary, our results demonstrate that mutation in Pcdh15 affects the initial formation of stereocilia bundles with associated changes in the actin meshwork within the cuticular plate; these effects are more pronounced in the presumed null mutation compared to mutations that only affect the extracellular domain. The positive correlation of severity of effects with extent of mutation can be seen well into adulthood.

Keywords: protocadherin 15, hair cell, cuticular plate, stereocilia

\section{INTRODUCTION}

Usher's syndrome type 1 (USH1) is a genetically heterogeneous group of recessive disorders that causes deafness in humans at or near birth and blindness by adolescence. Since the original report linking the Ames waltzer (av) mouse mutation to 
protocadherin 15 (Pcdh15) was published (Alagramam et al. 2001a), several mutations in the human ortholog of Pcdh15 have been linked to human syndromic (USH1F) and nonsyndromic (DFNB23) deafness (Ahmed et al. 2003; Alagramam et al. 2001a,b). More recently, in the Ashkenazi Jewish population, the R245X mutation of $\mathrm{PCDH} 15$ was reported to account for $58 \%$ of USH1 cases (Ben Yosef et al. 2003). These studies indicate the prevalence of congenital deafness associated with $\mathrm{PCDH15}$ mutation and the importance of understanding $P C D H 15$-associated inner ear pathology as it relates to the nature of the mutation. This knowledge will support optimal rehabilitation of communication in USH1F patients. However, it is difficult, if not impossible, to evaluate the onset and the course of pathology in patients carrying mutations in $\mathrm{PCDH} 15$ due to lack of appropriate tissue material. In mice, mutations in $P c d h 15^{a v-3 J}$ and $P c d h 15^{a v-T g}$ alleles of $a v$ result in premature stop codons. Similarly, two USH1F families with mutation in $\mathrm{PCDH} 15$ have been identified in which the mutations segregate with the disease phenotype and generate premature stop codons (Alagramam et al. 2001b). Therefore, mice carrying similar genetic lesions in Pcdh15 could serve as a model for inner ear pathology associated with USH1F and DFNB23.

Previously, we characterized different alleles of $a v$, including Pcdh15 $5^{a v-T g N 2742 R p w}, P c d h 15^{a v-3 J}, P c d h 15^{a v-2 J}$, and Pcdh15 ${ }^{a v-J}$ (Alagramam et al. 2000, 2001a,b) (Pcdh15 $5^{a v-T g N 2742 R p w}$ is written as Pcdh15 $5^{a v-T g}$ in this report). Pcdh15 is predicted to code for a transmembrane protein. Pcdh $15^{a v-J}$ and $P c d h 15^{a v-2 J}$ alleles carry in-frame deletions of the coding sequence and are considered to be less deleterious because these mutations would result in the absence of a small number of amino acids in the extracellular domain of the protein. In contrast, $P c d h 15^{a v-T g}$ and $P c d h 15^{a v-3 J}$ alleles are presumed to be functional null alleles because mutation in these alleles is predicted to code for a Pcdh15 protein lacking the entire transmembrane domain and all of the cytoplasmic domains.

In addition to being a model for inner ear pathology in USH1F and DFNB23 patients, av mice will help provide better understanding of the function of Pcdh15. In all av alleles studied thus far, no auditory evoked potentials can be recorded as early as they can be tested (P15) (Alagramam et al. 1999, 2001a) and all mutant mice display circling behavior by P10, indicative of vestibular dysfunction. This suggests that the mutation in Pcdh15 leads to early functional abnormalities of the mouse inner ear (Alagramam et al. 2005). Studying detailed ultrastructural development of the cochlear sensory epithelia in mutants and correlating that with the nature of the mutation among different alleles of a given mutation should provide additional information about the consequences of the loss of gene function.

In this study, the correlation between genotype and anatomic phenotype was investigated in four $a v$ alleles during postnatal development. Early changes in organ of Corti hair cell structure in mice aged $\mathrm{P} 0$ P10 were evaluated by electron microscopy. In addition, animals aged P15 to 12 months were studied by light microscopy to determine if the extent of mutation effects the long-term survival of inner ear structures outside the organ of Corti.

\section{METHODS}

Mice. The insertional mutant, $P c d h 15^{a v-T g}$, and methods for genotyping have been described previously (Alagramam et al. 1999). Pcdh15 $5^{a v J J}, P c d h 15^{a v-2 J}$, and $P c d h 15^{a v-3 J}$ alleles were originally obtained from The Jackson Laboratory and subsequently maintained as a breeding colony at Case Western Reserve University. For the all alleles, homozygous males were crossed to heterozygous females; the offspring from these matings were either heterozygous or homozygous for a specific allele. Mice homozygous for an av allele showed circling behavior by P10, compared to heterozygous littermates, which behaved similarly to wild-type mice. For mice younger than $\mathrm{P} 10$, a polymerase chain reaction (PCR)-based approach was used to genotype the Jackson alleles (described below). Protocol for animal use was approved by the Animal Care and Use Committee of the Case Western Reserve University.

Genotyping of Pcdh15 $5^{a v-J}, P c d h 15^{a v-2 J}$, and Pcdh15 $5^{a v-3 J}$ alleles. Mice were genotyped by PCR amplification from genomic tail DNA obtained from offspring derived from heterozygous by homozygous mating described above. Genomic DNA ( $\sim 500 \mathrm{ng} /$ reaction) was amplified under standard reaction conditions using Platinum Taq DNA polymerase (Invitrogen, Carlsbad, CA, USA) for 35 cycles of $94^{\circ} \mathrm{C}$ for $30 \mathrm{~s}, 55-58^{\circ} \mathrm{C}$ (depending on the primer pair used) for $30 \mathrm{~s}, 72^{\circ} \mathrm{C}$ for $30 \mathrm{~s}$. Primer pairs and product size are as follows: Pcdh $15^{a v-J}$ KA 15, $5^{\prime}$-TTC ACT ACA GCC TGG GGA AC-3' and KA 16 5'-GTT GAA TGT CGA GGG TGG AC-3' 156 bp product; Pcdh15 $5^{a v-2 J}$ KA26 5'-CTA GGG GAG GAC CAC CAG-3 ${ }^{\prime}$ and KA27 5'-CGT TTC GAC TCT CTT CAT CA-3', 72 bp product; Pcdh $15^{a v-3 J}$ KA $175^{\prime}$-GAC GGC AAA CTG CTC GAT A- $3^{\prime}$ and KA 18 5'-GGG ATG CAA CAG AGG ATG AT-3', 190 bp product. The annealing temperature for $\mathrm{KA} 15 / 16$ and $26 / 27$ is $55^{\circ} \mathrm{C}$; that annealing temperature for $\mathrm{KA} 17 / 18$ is $58^{\circ} \mathrm{C}$. It should be noted that the genotyping scheme for $a v-J$ and $a v-2 J$ is designed to distinguish heterozygous from homozygous offspring derived from crosses between heterozygote and homozygote parents. Primers KA 15/16 and KA 26/27 were designed to sequence DNA contained within the deleted area. For instance, when genotyping for the $a v-J$ allele, an amplified 
band of $156 \mathrm{bp}$ will indicate that the mice are "+/J" and the lack of amplified product (156 bp) band will indicate "J/J" genotype. As a positive control, $a v-2 J$ primers are added to the $a v-J$ genotyping reaction mix. Because $a v-J$ mice should carry a wild-type sequence at the "2J" site, the expected size (72 bp) product will be obtained. The same scheme is carried out when genotyping offspring derived from crosses between heterozygote and homozygote $a v-2 J$ parents. For the $a v-3 J$ allele, a different scheme is used. This takes advantage of the single nucleotide addition in the $a v-3 J$ allele (Alagramam et al. 2001a) that results in the introduction of a new restriction site, namely, MboII. The product from the wild-type DNA is $190 \mathrm{bp}$. Digestion of the 190-bp PCR product with MboII cleaves only the sequence harboring the $3 \mathrm{~J}$ mutation into 120- and 70-bp fragments, leaving the wild-type sequence undigested. With this approach, +/3J mice allele will show three bands (190, 120 , and $70 \mathrm{bp}$ ) and mice homozygous for the $3 \mathrm{~J}$ allele will show two bands (120 and $70 \mathrm{bp}$ ).

Histology. Inner ear tissue from Pcdh15 ${ }^{a v-J}, \operatorname{Pcdh} 15^{a v-2 J}$, Pcdh15 ${ }^{a v-3 J}$, and Pcdh15 ${ }^{a v-T g}$ homozygous and heterozygous mice at ages ranging from E18 to 12 months was studied. Tissue specimens were either processed, embedded in glycol methacrylate, then serial-sectioned for light microscopic study of the entire membranous labyrinth or prepared for transmission (TEM) and scanning electron microscopic (SEM) evaluation of the ultrastructure of the endolymphatic surface of organ of Corti.

All study animals were anesthetized and sacrificed by decapitation. The middle and inner ears were opened bilaterally, phosphate-buffered $2.5 \%$ glutaraldehyde fixative was flushed through the perilymphatic space, and the tissue was placed in fixative for 14-18 $\mathrm{h}$ at $4^{\circ} \mathrm{C}$ (Alagramam et al. 2000). Tissue was then rinsed in $0.1 \mathrm{M}$ sodium phosphate buffer $(\mathrm{pH}$ 7.4-7.5) and stored at $4^{\circ} \mathrm{C}$ for several days prior to processing for light and electron microscopic viewing.

Specimens from P15 to 12-month Pcdh15 $15^{\text {av-J }}$, Pcdh15 ${ }^{a v-2 J}, P c d h 15^{a v-3 J}$, and Pcdh15 $5^{a v-T g}$ homozygous and heterozygous mice were prepared for light microscopic study to examine changes in the inner ear structures. Preparation of material for light microscopic examination was as follows: Temporal bones were dissected from the skull and decalcified in $0.35 \mathrm{M}$ EDTA for two to four days at room temperature with agitation, rinsed in saline, dehydrated in a series of alcohols, infiltrated and embedded in glycol methacrylate (JB-4, Polysciences, Warrington, PA, USA) embedding solution. Serial sections at a thickness of 3-5 $\mu \mathrm{m}$ were taken in a plane approximately parallel to the long axis of the modiolus, and every tenth section was stained with toluidine blue, coverslipped, and viewed using a compound microscope. Analysis of each set of sections included the entire cochlea and all vestibular sensory organs.

Organ of Corti samples from E18 to P10 homozygous and heterozygous mice were prepared for either SEM or TEM to examine ultrastructural changes in the cuticular plate. Glutaraldehyde-fixed and bufferrinsed inner ear samples were stained with 1-1.5\% osmium tetroxide, followed by a buffer rinse. Material to be studied by TEM was then decalcified in 0.35 M EDTA, rinsed in buffer, dehydrated in a graded series of ethanols, and embedded in Spurrs resin. The basal turn of the organ of Corti was thinsectioned either in a plane parallel to the surface of the reticular lamina or in a plane perpendicular to that surface and radial to the modiolus. Sections were then stained with lead citrate and uranyl acetate and viewed using a JEOL 1200EX transmission electron microscope. For SEM, the organs of Corti were exposed via cochlear microdissection and the tissue was dehydrated in a series of ethanols, critical point dried, coated with gold-palladium, and studied using a JEOL 848 scanning electron microscope.

\section{RESULTS}

Light and electron microscopic examination of the four $a v$ alleles included in this study (see Fig. 1 for deletion sites) revealed a clear difference in the severity of alterations in the organ of Corti depend-

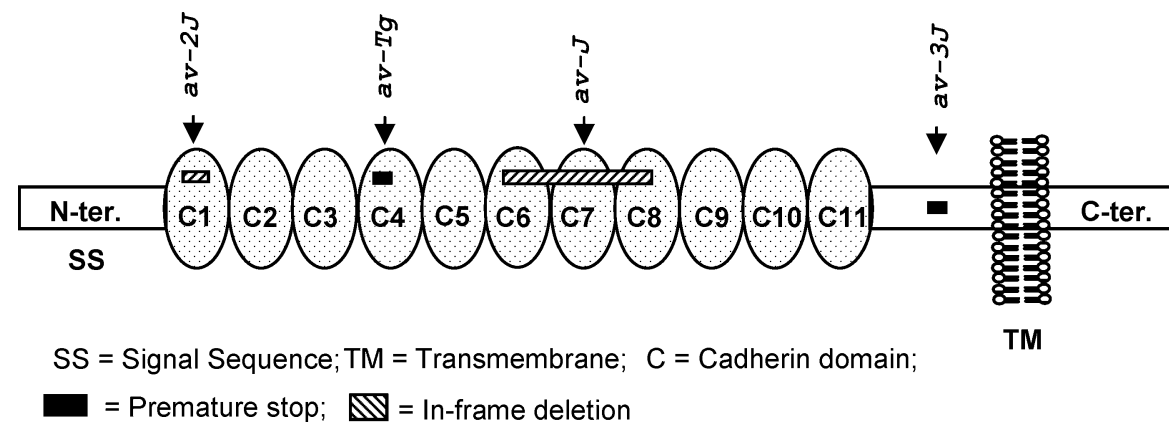

FIG. 1. Mutation in the four alleles of Pcdh15 used in this study. 
ing on the extent of Pcdh15 mutation. Electron microscopic examination of specimens from immature mice showed a more severe phenotype in animals harboring the functional null mutations than in mice harboring in-frame deletion mutations. In addition, light microscopic study of mice ages P15 up to 12 months revealed that the extent of mutation affects the long-term survival of inner ear structures outside the organ of Corti.

\section{Scanning electron microscopic findings in E18-P10 mice}

No changes in inner ear morphology were detected by light microscopy in temporal bone cross sections from homozygous Pcdh15 mutants at P10 or earlier. This section focuses on electron microscopic findings from mice aged E18-P10 (19 heterozygous controls, 19 presumed functional nulls, 17 in-frame deletions). Light microscopic findings from animals aged 15 days or older are presented in subsequent sections.

Abnormalities in the organ of Corti were observed via SEM in all homozygous mice studied at ages E18P10 (Figs. 2 and 3). Although the most obvious defects were in the outer hair cells (OHCs), the inner hair cells (IHCs) were also affected. The flattened U-shape arrangement of stereocilia on the inner hair cell cuticular plate was close to normal in all animals of all mutations at P10. In the most affected inner hair cells, the arrangement of stereocilia resembled a wavy line more than a "U," with

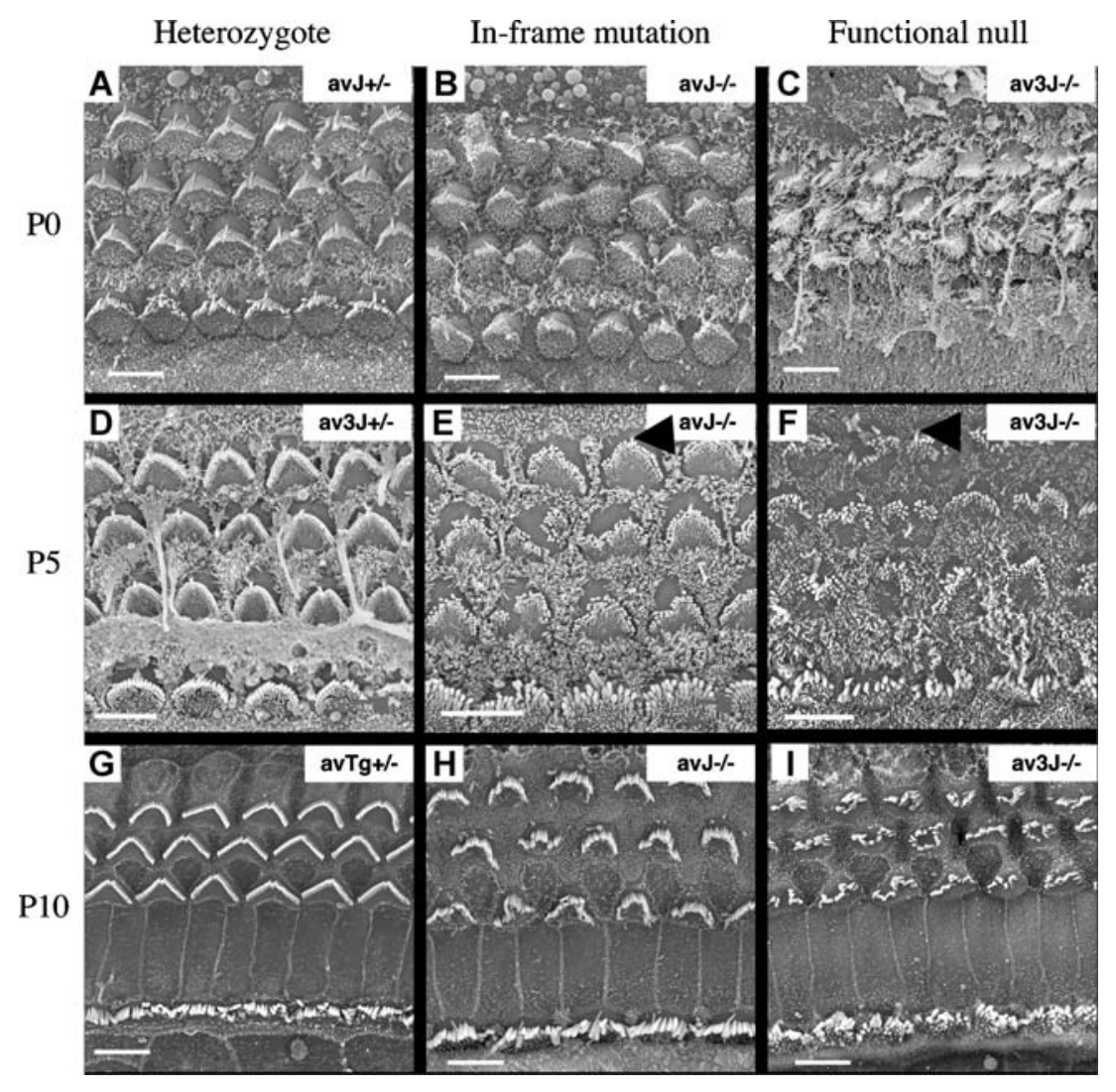

FIG. 2. Scanning electron micrographs of the surface of the organ of Corti in immature mice aged P0 (A-C), P5 (D-F), and P10 (G-I). The normal arrangement of stereocilia and kinocillia can be seen on the surface of the inner hair cells (IHCs, bottom row) and three rows of outer hair cells (OHCs, upper three rows) in tissues from heterozygous control mice (A, D, and G). The outline of the hair cells at the surface of the organ of Corti changes from a circular shape at $\mathrm{P0}$ (A) to a triangular shape by $\mathrm{P} 5$ for $\mathrm{OHCs}$ (D) and an oval shape by $\mathrm{P} 10$ for IHCs (G). A more severe phenotype can be seen in the organs of Corti from mice with presumed functional null mutations $(C, F$, and $\mathrm{I})$ than in in-frame deletions $(B, F$, and $H)$. The $\mathrm{OHC}$ stereocilia bundles are more disorganized, and kinocilia placement is more affected ( $F$ arrow) compared to mice with in- frame deletions (E arrow). The stereocilia bundles of both the $\mathrm{OHCs}$ and IHCs are disorganized by $\mathrm{PO}$ (compare $\mathrm{C}$ to B), but the extent of displacement is greater for $\mathrm{OHCs}$ and it becomes more apparent as the surface of the cells matures ( $\mathrm{F}$ and $\mathrm{I})$. The outline of the surface of OHCs is abnormal in all three rows in Pcdh $15^{a v-31}$ mice by P5 (F), whereas the IHC outline is relatively unaffected up to P10 (I). Some alterations in the arrangement of the stereocilia and the position of the kinocillia can be seen at P0 in Pcdh15 $5^{\text {av- }}$ mice. By P5, the abnormal arrangement of stereocilia becomes more apparent (E) and an abnormal shape of the cuticular plate is seen $P c d h 15^{a v-J}$ by $\mathrm{P} 10$ $(\mathrm{H})$. The arrangement of stereocilia on IHCs and the cell outline are less affected than that of OHCs. Bar $=5 \mu \mathrm{m}$. 


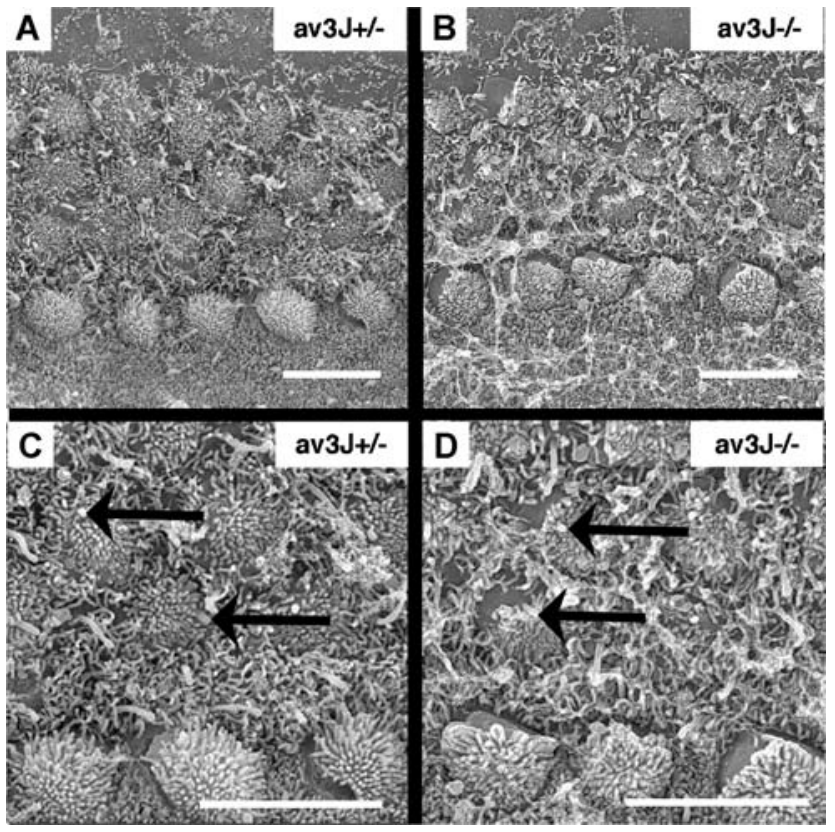

FIG. 3. Scanning electron micrographs of the surface of the organ of Corti in immature mice aged E18. Microvilli and stereocilia cover the surface of the hair cells in heterozygous controls (A). Bare areas of the cell surface can be seen in many of the hair cells in PCdh1 $15^{\text {av-3) }}$ mice (B, D) with accompanying disorganization of stereocilia and microvilli. (C and D) Higher magnification view of $\mathbf{A}$ and $\mathbf{B}$, respectively. At this age, kinocillia (arrows) can be located either in the center or at the periphery of the cell in either the control or the mutant mice. Bar $=5 \mu \mathrm{m}$.

some splaying of the stereocilia above the point of insertion into the cell (Fig. 2I). The changes in outer hair cell surface ultrastructure were analyzed by extent of mutation and age.

Presumptive functional null alleles. Changes in outer hair cell (OHC) morphology of the functional null alleles were more severe than the in-frame deletion alleles at ages P0-P10 (Fig. 2). The majority of the outer hair cells were abnormal in all the ears examined from the $P c d h 15^{a v-T g}$ and $P c d h 15^{a v-3 J}$ alleles at this age, with the exception of one ear from a mouse with the $P c d h 15^{a v-3 J}$ mutation. This ear had a large area near the base of the organ of Corti with normal "W"-shaped arrangement of stereocilia on the surface of the OHCs. Pcdh1 $15^{a v-T g}$ and Pcdh1 $15^{a v-3 J}$ show near-identical results; only data from $P c d h 15^{a v-3 J}$ are shown here.

E18 ears from heterozygous control and $P c d h 15^{a v-3 J}$ mice were examined to determine the earliest developmental stage at which abnormalities in the apical surfaces of the hair cells could be observed. In several specimens, differentiation had not proceeded far enough to permit reliable interpretation. However, organs of Corti from three control and three homozygous mice proved mature enough to visualize both inner and outer hair cell surfaces. Examination of these hair cells revealed abnormalities in the mutants at a time when stereocilia are visible in these tissues by SEM (E18; Fig. 3). The surfaces of inner and outer hair cells from control mice were covered with stereocilia and microvilli (Fig. 3A and C). Kinocilia could be seen either in the center or at the periphery of hair cells from both control and mutant mice (arrows, Fig. 3C and D). Alterations observed in the functional null mice at E18 included disorganization of stereocilia and microvilli and the presence of large bare areas on the surfaces of hair cells. Also, the cell surface sometimes had an irregular, rather than round shape (Fig. 3B and D).

Several of the $P c d h 15^{a v-3 J}$ ears examined from E18 (Fig. 3D) and ears from P5 (Fig. 2F) animals showed no reduction in the number of stereocilia or microvilli. In P10 mice with functional null mutations, alterations in OHC stereocilia arrangement varied from slight deviations in the "W" shape with a normal compliment of stereocilia, to tilted "W," to clumping stereocilia bundles, or "C"- and "O"-shaped arrangements of stereocilia with a reduction in the number of short stereocilia. This reduction in short stereocilia appears to be attributable to a degradation in the condition of cuticular plates at this stage (P10). Fusion of the stereocilia and bulges on the cuticular plate were not seen in ears from these mutants by P10 (Fig. 2).

In-frame deletions. Mice harboring in-frame deletions Pchd15 $5^{a v-J}$ and Pcdh15 $5^{a v-2 J}$ showed varying degrees of organ of Corti pathology. The Pcdh $15^{a v J}$ specimens were the least affected of the alleles studied. Less than half of the outer hair cells appeared to be abnormal with the extent of pathology varying between animals $(\mathrm{P} 0-\mathrm{P} 10)$. At $\mathrm{P} 0$, the most common anomaly seen via SEM in the outer hair cells of mice carrying the inframe deletion mutation was a flattening of the "W"shaped arrangement of the stereocilia on the cuticular plate (Fig. 2B). In some cases, the cuticular plate also appeared markedly rotated; with the kinocilium located in the 10 o'clock or 2 o'clock position instead of the normal 12 o'clock position. This rotation occurred regardless of whether the "W"-shaped arrangement of the stereocilia continued to orient to the kinocilia. At P5 (Fig. 2E), in cells on which the typical arrangement of stereocilia was disrupted, there was still some organization in the rows of stereocilia of $P c d h 15^{a v-J}$ and $P c d h 15^{a v-2 J}$ mice such that the height of the rows was maintained short to tall. Cell surfaces containing only scattered clumps of stereocilia were rarely seen. A similar, but slightly more severe, effect was observed in $P c d h 15^{a v-2 J}$ mice (data not shown). Cells on which the stereocilia were shaped more like an "O" than a "W" were also rare in the $P c d h 15^{a v-J}$ and $P c d h 15^{a v-2 J}$ mice. Even in the most affected cells, a full compliment of stereocilia could usually be seen in ears from Pchd15 $5^{a v-J}$ mutants up to P10 (not shown). There was some reduction in numbers of the shorter stereocilia in the 
more affected of these cells, but fusion of the stereocilia and bulges on the cuticular plate were not seen.

\section{Transmission electron microscopic findings in P0-P10 mice}

Cochlear tissues from heterozygous and homozygous $P c d h 15^{a v-J}, P c d h 15^{a v-2 J}, P c d h 15^{a v-3 J}$, and Pcdh15 $5^{a v-T g}$ animals aged P0-P10 were examined by TEM for study of the hair cell cuticular plates (20 heterozygous controls, 13 presumed functional nulls, 17 inframe deletions). The cuticular plate is immature at this age, with a less well-defined structure compared to adult (Figs. 4 and 5). However, the basal body, rootlets of the stereocilia, developing cell junctions, and the developing actin meshwork within the

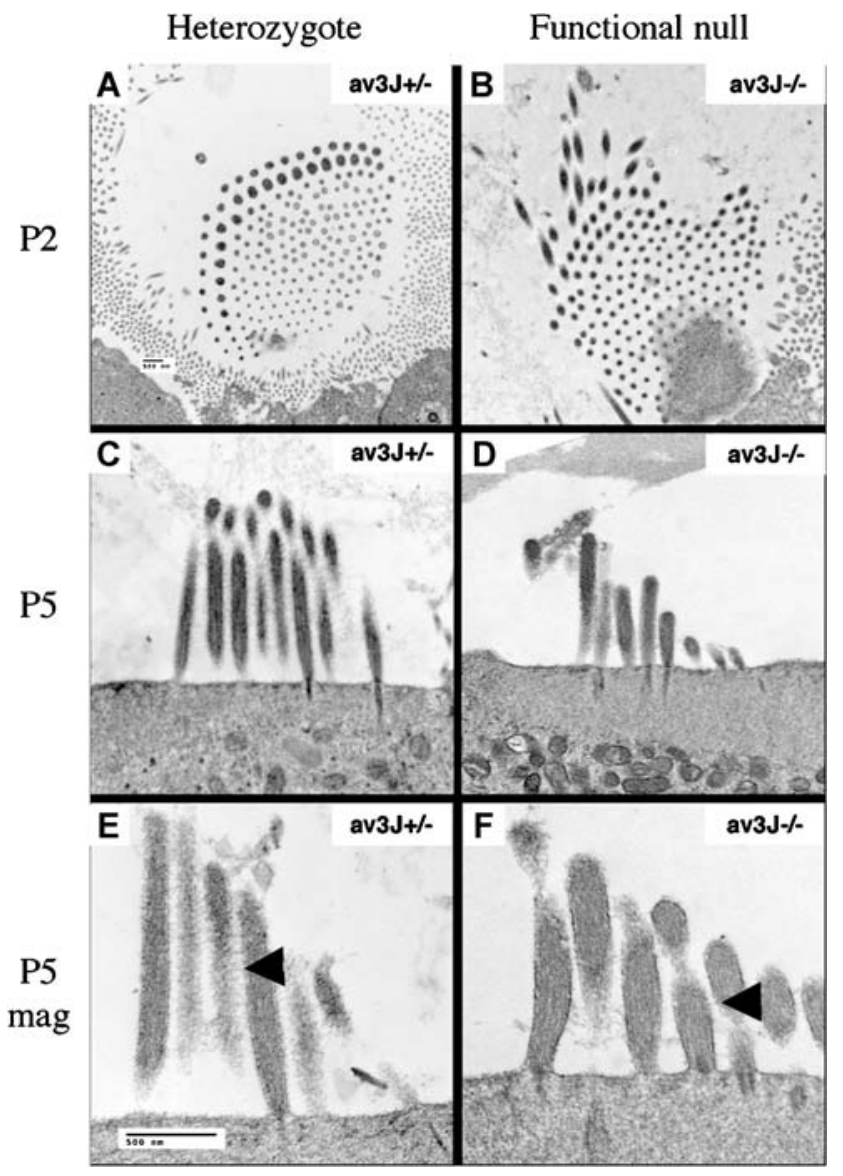

FIG. 4. Transmission electron micrographs of sections through the hair cells in immature hair cells in heterozygous, control, and functional null mutant mice. (A, B) Cross sections through the surface of the inner hair cells of mice aged P2 showing some derangement in the cell from the functional null mutant. (C-F) Longitudinal sections through the cuticular plate and stereocilia bundle of P5 heterozygous and functional null mutant mice. (E, F) Higher magnification views from two other representative cells. No clear ultrastructural differences were found using this method of observation. Lateral links can be seen in both samples (E, $\mathbf{F}$ arrows). cuticular plate could be visualized in both inner and outer hair cells by $\mathrm{P} 2$ and are fairly prominent by P10 (Fig. 5G-L). Inner hair cell stereocilia of heterozygous control ears at $\mathrm{P} 2$ were already arranged in a flattened "U" shape, oriented to the kinocilia and the lateral aspect of the cuticular plate (Fig. 4A). Inner hair cell cuticular plates in all the homozygous specimens showed disorganization of the stereocilia (Fig. 4B). The arrangement of outer hair cell stereocilia is quite abnormal in $P c d h 15^{a v-3 J}$ mice at $\mathrm{P} 0$ by SEM, but no ultrastructural changes were seen by TEM in cells sectioned perpendicular to the surface of the reticular lamina (Fig. 4C-F). No abnormalities in stereocilia length, thickness, rootlet structure or cuticular plate density were seen in mice as old as P5. Although this material was not fixed in a manner optimum for visualization for lateral links, they appear to be present in cells from both the control and $P c d h 15^{a v-3 J}$ mice (arrows, Fig. $4 \mathrm{E}$ and F). Because of the inability to identify changes by observation with this orientation, majority of TEM data for this study were analyzed in the cross-sectional plane, parallel to the surface of the reticular lamina.

When viewed in cross section, changes were apparent by P0. Outer hair cell stereocilia of control ears at P0 had formed a partial "W"-shaped arrangement at the surface of the cell, which was oriented to the basal body, from which the kinocilium extends during development (Fig. 5A). This shape is more evident by $\mathrm{P} 2$ (Fig. 5D), and by $\mathrm{P} 10$ the mature stereocilia arrangement was seen on the outer hair cell surface (Fig. 5G), along with a fairly well developed mesh within the cuticular plate (Fig. 5J arrow). Cross sections of homozygous outer hair cells showed the disorganization of stereocilia seen in the SEM samples of ears from mice of the same age and genotype. The arrangement of stereocilia in Pcdh15 av$J$ and $P c d h 15^{a v-2 J}$ mice at P0 was not markedly different from controls, typically showing only a slight disorganization in the rows of stereocilia (Fig. 5B). Stereocilia on the outer hair cell surface in mice carrying a functional null mutation showed a more dramatic disorganization of stereocilia on hair cells at this early stage of development (Fig. 5C). This disorganization of the stereocilia became more evident as the cells matured (Fig. 5F and I). In addition to changes in stereocilia, several alterations were seen in the cuticular plate itself. The rootlets of the stereocilia were sometimes broken (arrowhead, Fig. 5L) and the dense-staining actin mesh within the cuticular plate disorganized (Fig. 5I and L). In mice carrying functional null mutations, the cuticular plate appeared markedly rotated; with the basal body of the kinocilium located in the 10 o'clock or 2 o'clock position instead of the normal 12 o'clock position (Fig. 5F and I, arrows). This rotation occurred 
regardless of whether the "W"-shaped arrangement of stereocilia continued to orient to the kinocilia. In some extreme cases, the basal bodies were on the opposite side of the cell and several cells had the poles of the basal body lying parallel to each other instead of the normal perpendicular arrangement. This was also seen in some of the $P c d h 15^{a v-J}$ and Pcdh15 ${ }^{a v-2 J}$ mice, but much less often.

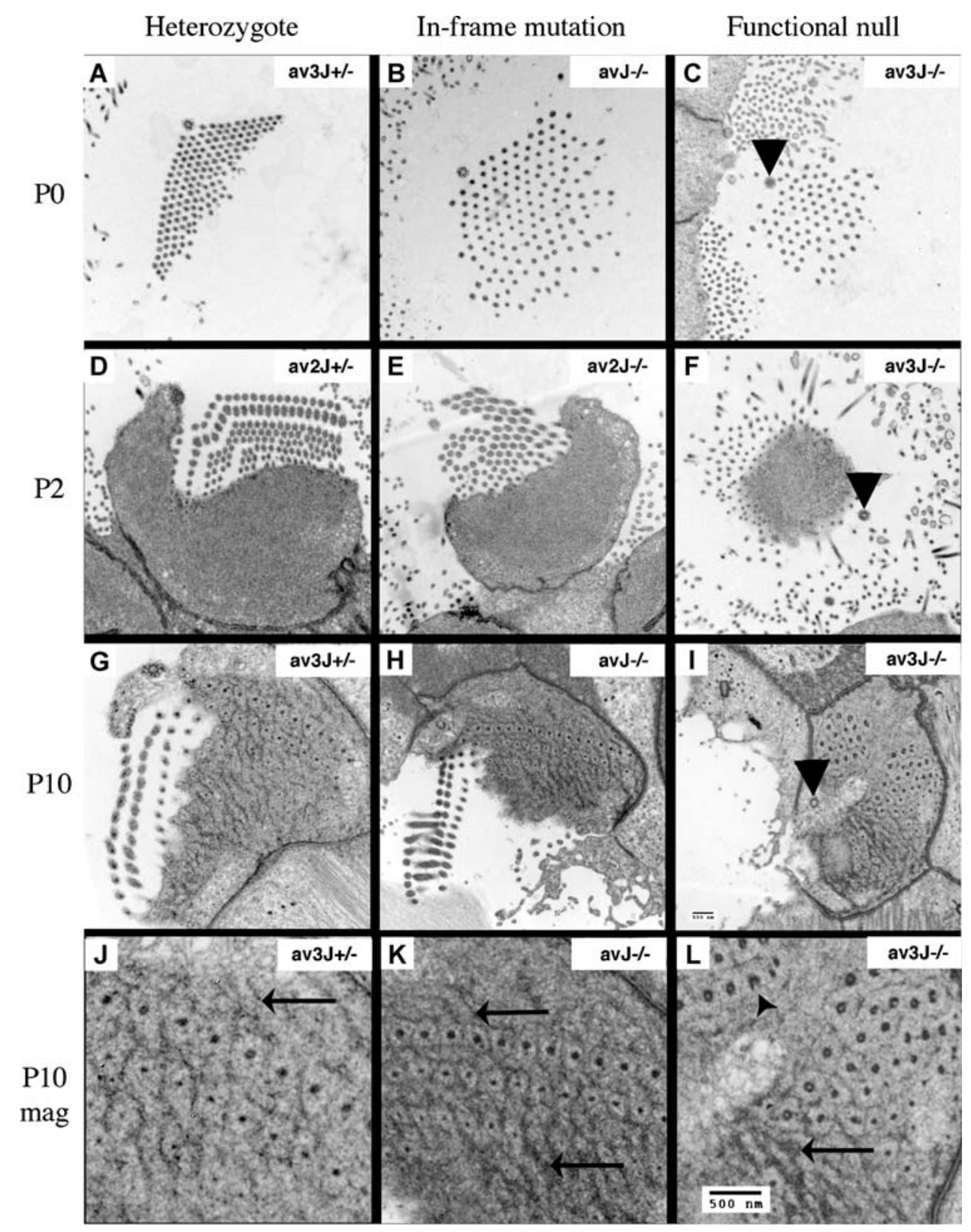

FIG. 5. Transmission electron micrographs of cross sections through the surface of the organ of Corti in immature mice aged P0 (A, B, and C), P2 (D, E, and F) and P10 $(\mathbf{G}-\mathbf{L})$ near the level of insertion of the stereocilia into the cuticular plate. Normal arrangement of stereocilia and kinocilia can be seen in outer hair cells at P0 (A), P2 (D), and P10 (G) from heterozygous control mice. A dense staining mesh within the actin of the cuticular plate can be seen surrounding the stereocilia and kinocilia in the samples from heterozygous and in-frame deletion mice at P10 ( $\mathbf{J}, \mathbf{K}$ and $\mathbf{L}$ arrows). The actin mesh within the cuticular plate is not as well developed by P2 (D and $\mathbf{E}$ arrows). (B, E, and $\mathbf{H}$ ) Tissue from homozygous mice with in-frame deletions in Pcdh15. A slight derangement can be seen in the rows of stereocilia near the surface of the cuticular plate at P0 (B) and P2 (E). The P10 specimen appears fairly normal, which was often seen in this group $(\mathbf{H})$. (C, $\mathbf{F}$ and $\mathbf{I})$ Tissue from homozygous mice with presumed functional null mutations in Pcdh15. The stereocilia near the surface are displaced to a greater degree than those seen in the tissue from the in-frame deletion. The rows of stereocilia on the surface of OHCs are frequently significantly disorganized in this group; often the kinocilium is also out of position (F and $\mathbf{I}$ arrows). At P10 (I), the actin mesh is present but out of place, still oriented to the abnormal positions of the stereocilia and kinocilium. $\mathbf{I}$ and $\mathbf{L}$ Bar $=500 \mathrm{~nm}$. 
Changes in the insertion patterns of stereocilia into the cuticular plate of the inner hair cells could be seen in specimens from homozygous animals by P0 (not shown). Otherwise, no other abnormalities could be seen by TEM in the inner hair cell apices of mutant mice from $\mathrm{P} 0$ to $\mathrm{P} 10$.

\section{Light microscopic findings, progression} of pathology from P15 to 12 months

Organ of Corti pathology in homozygous mice becomes severe enough to be obvious by light microscopy at time points of P15 and older. This section describes light microscopic findings relating to the progression of pathology from loss of outer hair cells to loss of spiral ganglion cells and alterations in other inner ear tissues from mice aged 15 days and older.
In heterozygous control mice $(N=11)$, a slightly short appearance was noted in the basal turn outer hair cells in two of the ears from mice aged P15-P20 and two of the ears from mice aged P25 had smallappearing spiral ganglion cells at the base of the modiolus. All other ears had normal-appearing organs of Corti and spiral ganglion cells for their stage of development. The spiral ligament, spiral limbus, stria vascularis, and vestibular apparatus appeared normal in all animals of this group (Fig. 6A, B, and C).

Conversely, inner ear specimens from homozygous mice at age 15-25 days showed organ of Corti pathology with variation in the degree of pathology depending on the allele. The alleles with in-frame deletions were less affected than the functional null alleles. The morphologic changes are as follows.

Presumptive functional null alleles (P15-P25, $N=15)$. Moderate organ of Corti pathology was seen in both Pcdh15 $5^{a v-3 J}$ and Pcdh15 $5^{a v-T g}$ mutants (Fig. 6G) with

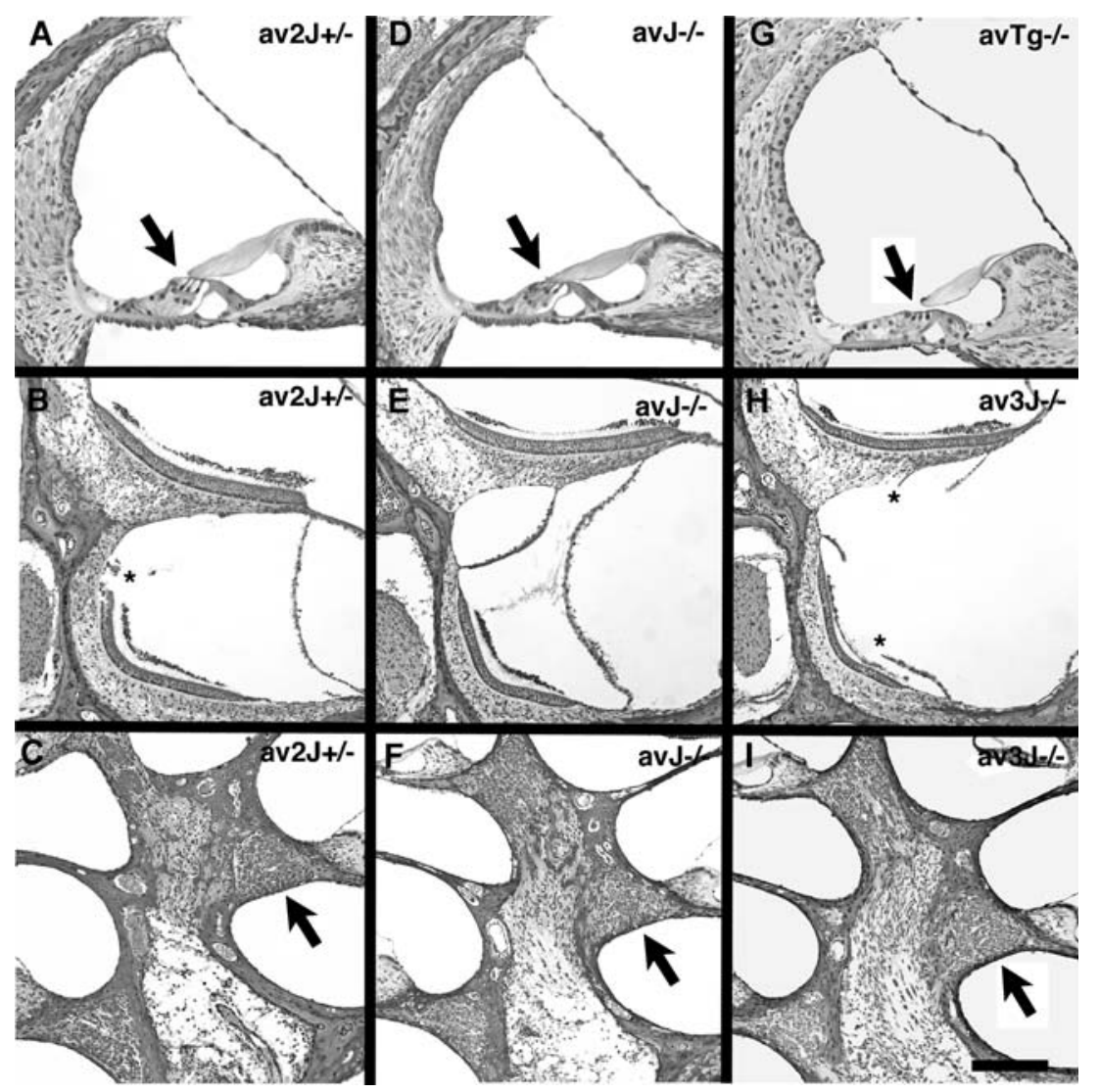

FIG. 6. Light micrographs of cross sections of P15 mouse inner ears. Normal morphology can be seen in scala media cross sections from midbasal turns of cochleae from heterozygous control mice (A) and from mice homozygous for an in-frame deletion of Pcdh15 (D). Swelling of the supporting cells and shortening of outer hair cells with loss of the spaces of Nuel are seen in cross sections from mice homozygous for a presumed functional null mutation (G, arrow). Normal morphology can be seen in cross sections of the utricular (top) and saccular (bottom) maculi in control mice (B), mice homozygous for in-frame deletions (E), and mice homozygous for a presumed functional null mutations in Pcdh15 (H) at this age. A normal compliment of slightly immature spiral ganglion cells can be seen in cross sections of the modiolus of control mice $(\mathbf{C})$, mice homozygous for in-frame deletions (F), and mice homozygous for presumed functional null mutations in Pcdh15 (I). Bar $=0.1 \mathrm{~mm}$ for A, D, G, and $0.2 \mathrm{~mm}$ for rest. Arrows point to upper basal spiral ganglion; ${ }^{*}=$ artifact. 
minimal spiral ganglion changes (Fig. 6I). Changes included patches of organ of Corti damage with inner and outer hair cell loss and collapse, which was seen from base to apex with some associated loss of spiral ganglion cells in the base only. Pathologic changes in the lateral wall, the spiral limbus, or the vestibular apparatus in this age group were limited to a slight thinning of fibrocytes in the apical part of the spiral ligament from one ear and a thinning of the stria vascularis in the apical part of a different ear. Most of the lateral wall tissues, the spiral limbus, and the vestibular apparatus from homozygous animals at this age appeared normal (Fig. 6G-I).

In-frame deletions (P15-P25, $\mathbf{N}=\mathbf{1 1})$. The degree of pathology seen in these specimens ranged from minimal organ of Corti pathology (limited to a shortening or loss of outer hair cells and Deiters' cell swelling at the base of the cochlea with no spiral ganglion changes) in Pcdh15 $5^{a v-J}$ mutants (Fig. 6D and F) to mild/moderate organ of Corti pathology (outer and inner hair cell loss, Deiters' cell swelling, and collapsed tunnel of Corti in the base with some associated loss of spiral ganglion cells) in Pcdh15 $5^{a v-2 J}$ mutants. The spiral ligament, spiral limbus, stria vascularis, and vestibular apparatus appear normal in all homozygous animals of this group (Fig. 6D and E).

\section{Light microscopic findings in adult mice}

Inner ears from P30 to P50 heterozygous control mice $(N=4)$ appeared normal. Inner ear specimens from heterozygous control mice aged 6.5 months $(N=2)$, showed moderate inner ear alterations (Fig. 7A-C). Scattered loss of outer hair cells was seen at the base of the cochlea with some thinning of spiral limbus fibrocytes and loss of spiral ganglion cells in the basal portion of the cochlea. More than three quarters of the spiral ganglion cells remained intact, with occasional patchy loss and clumping of the cell bodies in the modiolar apex. Occasional thinning of the fibrocytes in the spiral limbus was also seen at the apex of some cochleae. These changes could be a related to aging in the 6.5-month-old ears because they have a C57BL/6J background (Francis et al. 2003; Ichimiya et al. 2000). All control ears had normalappearing vestibular neuroepithelia (Fig. 7B).

Changes were observed in all alleles of homozygous mice studied by 30-50 days of age (data not shown). By this age, changes in homozygous mice were more pronounced than those in older control ears at age 6.5 months. The pathology in $P c d h 15^{a v-J}$ mutants at P50 $(N=3)$ included complete loss of outer hair cells in the base to patchy loss in the apex. The basal portion of the spiral ganglion had a reduction in cell number by approximately one fifth. No pathology was seen in the spiral limbus, stria vascularis, or spiral limbus. Degenerating otoconia were occasionally found overlying the saccular neuroepithelia. However, no significant changes in the morphology of hair cells were observed in the saccule. The neuroepithelia of the utricle or semicircular canals appeared normal. Some of these findings were reported earlier (Alagramam et al. 2005).

One $P c d h 15^{a v-3 J}$ mutant and one $P c d h 15^{a v-2 J}$ mutant showed cuboidal cells replacing the cells of the organ of Corti at the base with collapse of the tunnel of Corti in apical regions. Less than half of spiral ganglion cells remained in the modiolar base with a smaller loss (approximately 20\%) in the apex. No pathology was seen in the spiral ligament, stria vascularis, or spiral limbus. Only the condition of the saccular neuroepithelia differed between these two ears, with more changes in saccular otoconia and more hair cell loss seen in the functional null $\left(P c d h 15^{a v-3}\right)$ animal. No changes were observed in any of the other vestibular neuroepithelia of P50 mice.

Homozygous alleles ranging in age from 6.5 to 12 months showed a considerable amount of inner ear pathology; however, the degree of pathology seen in the ears of in-frame deletion $\left(P c d h 15^{a v-J}\right)$ mice appeared to be less than that observed in the ears from functional null alleles $P c d h 15^{a v-3 J}$ and $P c d h 15^{a v-T g}$ (Fig. 7D-I). The results are as follows.

Presumptive functional null alleles (6.5-12 months, $N=7$ ). Inner ear specimens from homozygous $P c d h 15^{a v-T g}$ and $P c d h 15^{a v-3 J}$ mice, ranging in age from 7 to 12 months, showed a consistent, dramatic loss of organ of Corti with cuboidal-cell scar formation in the basal turn (Fig. $7 \mathrm{G}$ ), plus patchy scarring and almost complete loss of outer hair cells throughout the rest of the cochlea. Atrophy of spiral ligament fibrocytes was consistently seen in the base of the cochlea, sometimes extending into the apex. Atrophy of spiral limbus fibrocytes was consistently limited to the apical region. Less than one tenth of the spiral ganglion cell bodies remained (Fig. 7I) in any of the seven cochleae examined. Patches of strial atrophy were also present in five of seven ears examined. In these ears, very few hair cells remained in the saccular macula (Fig. $7 \mathrm{H}$ ). There was also obvious vestibular ganglion cell loss in the area of saccular neuroepithelium, whereas the other vestibular neuroepithelia appeared normal by light microscopy.

In-frame deletions (6.5-8 months, $N=7$ ). Ears from the group of Pcdh15 av-J mice showed organ of Corti damage ranging from loss of the tunnel of Corti in the base with areas in the apex more or less intact (three of seven ears) to a cuboidal-cell scar formation in the base of the cochlea with inner hair cells and supporting cells remaining in the apex (four of seven ears). Spiral ganglion cell loss ranged from less than one tenth of cells remaining (two of seven ears, Fig. $7 F$ ) to more than three quarters of the cells 


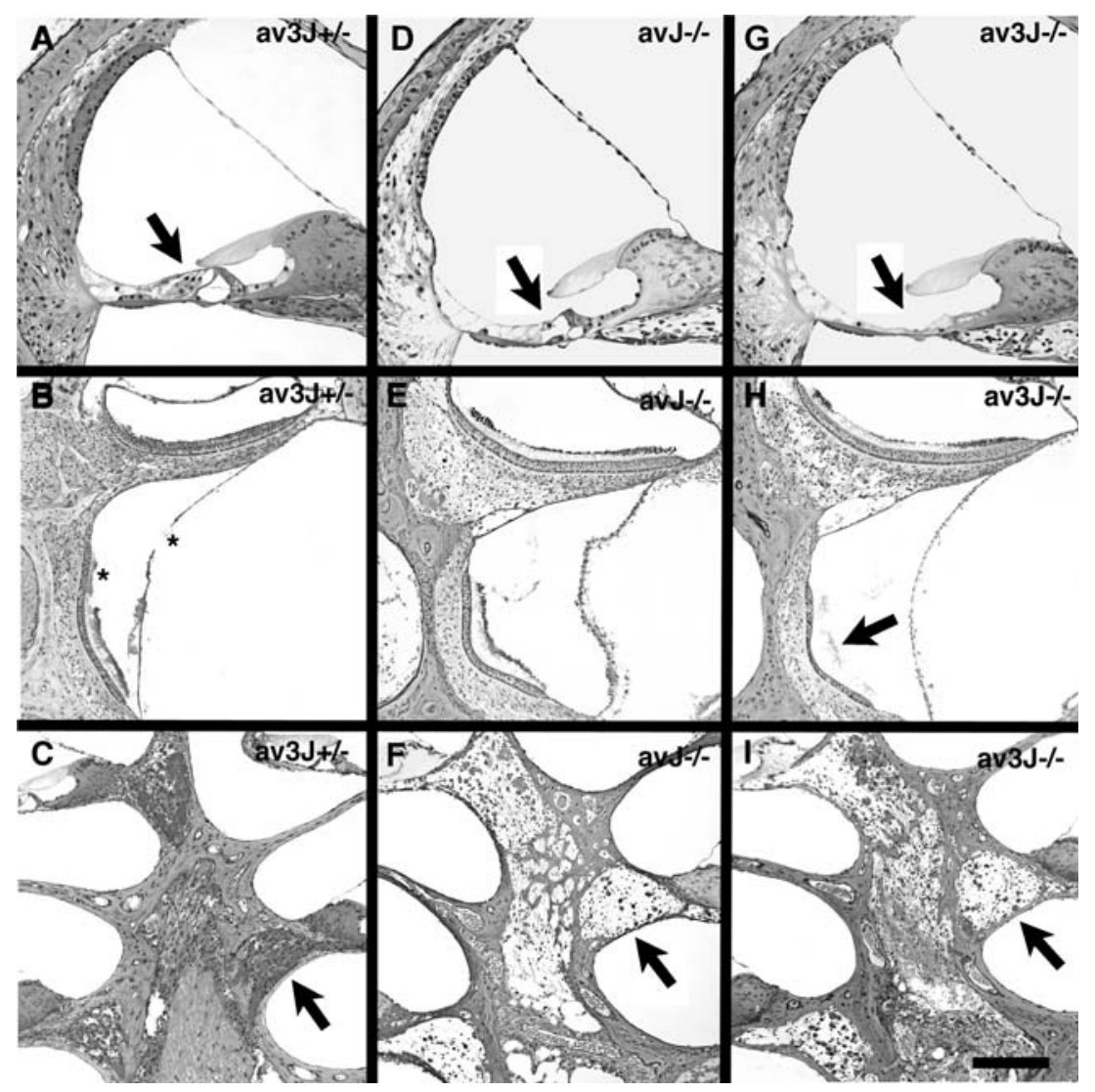

FIG. 7. Light micrographs of cross sections of inner ears from 6.5month-old mice. Normal morphology can be seen in scala media cross sections from the midbasal region of the cochlea $(\mathbf{A}$, arrow $=$ organ of Corti), sections from the utricular (B, top) and saccular (B, bottom) maculi, and from the spiral ganglion (C) of ears from heterozygous control mice. Damage can be seen in the lateral wall, nerve fibers, and organs of Corti (D and $\mathbf{G}$ arrows) in cross sections from mice homozygous for in-frame deletions (D) or homozygous for presumptive functional null mutations in $\operatorname{Pcdh} 15$ (G), with a simple cuboidal epithelium often replacing the organ of Corti in the functional null group. Normal morphology can be seen in the utricular (E, top) and saccular (E, bottom) maculi from mice carrying

remaining in two of the oldest animals examined. Atrophy of spiral ligament fibrocytes was often seen (Fig. 7D) with more thinning at the base of the cochlea. On the other hand, atrophy of the spiral limbus fibrocytes was restricted to the apex in all cochleae examined in this group. Vestibular changes were limited to degeneration of otoconia in the saccule. No reduction in saccular hair cells was observed in any of these ears. No changes were observed in any other vestibular neuroepithelia (Fig. 7E).

\section{DISCUSSION}

This study focused on morphological alterations of the cochlea and vestibular apparatus in mice harbor- the in-frame deletions. In the mice carrying the presumed functional null mutation $(\mathbf{H})$, hair cell loss can be seen in areas of the saccular macula with partial or complete loss of saccular otoconia (arrow), whereas the morphology of the utricular macula is normal. Spiral ganglion cross sections from control ears $(\mathbf{C})$ look normal from the midbasal turn (arrow) to apex, with only slight cell loss in the far basal end of the cochlea. Both mice homozygous for in-frame deletions in (F) and mice homozygous for presumptive null mutations in Pcdh15 (I) have lost the majority of their spiral ganglion cells at this age. Bar $=0.1 \mathrm{~mm}$ for $\mathbf{A}, \mathbf{D}, \mathbf{G}$, and $0.2 \mathrm{~mm}$ for rest; ${ }^{*}=$ artifact.

ing mutations in Pcdh15. Inner ear tissues from animals ranging in age from embryonic day 18 to 1 year were examined by light and electron microscopy. It was found that the degree of pathology correlates with the extent of mutation in Pcdh15 in all four alleles included in the investigation. This finding lends support to the contention that the inner ear changes occurring over time in Usher syndrome type $1 \mathrm{~F}$ are a direct consequence of the mutation in $\mathrm{PCDH} 15$. By studying several alleles and evaluating cellular and subcellular changes that correlate with the severity of the mutation, we can better determine the role of Pcdh15 in the development and maintenance of the inner ear, the organ of Corti in particular.

Of the alleles we studied, the Pcdh $15^{a v-J}$ mutants consistently showed the smallest degree of inner ear pathology. Pcdh15 $5^{a v-2 J}$ mutants, on the other hand, 
displayed large variations in degree of pathology. The two presumptive functional null mutants, Pcdh $15^{a v-3 J}$ and Pcdh15 $5^{a v-T g}$, showed severe phenotypes that were very similar to one another with regard to severity of inner ear pathology. These animals showed markedly more severe sensory cell changes early in development, which consistently progressed more rapidly than those observed in the in-frame mutants.

The first cadherin domain is believed to be important for the function of protocadherins (Suzuki 2000). Data presented here provide some support for that notion. The $P c d h 15^{a v-2 J}$ allele is predicted to have 27 amino acids deleted from the first cadherin domain compared to 85 amino acids predicted to be deleted from the 6 th, 7 th, and 8 th cadherin domains in the $P c d h 15^{a v-J}$ allele. However, inner ear tissues from animals carrying the Pcdh $15^{a v-J}$ mutation tend to be less severely affected than those from Pcdh15 $5^{a v-2 J}$ mutants. Thus, mutations in the first cadherin domain result in a more severe phenotype, which supports the contention that the first domain is an important functional component of Pcdh15.

The specific functions of Pcdh15 in the inner ear are not fully understood. The cochlear abnormalities observed to date in Pcdh15 mutants strongly suggest that Pcdh15 is important for the normal development of stereocilia and the cuticular plate. However, it was recently demonstrated that animals carrying presumptive functional null mutations in Pcdh15 show a complete lack of peripheral vestibular function even though stereocilia abnormalities comparable to those in the cochlea are not present in vestibular hair cells (Alagramam et al. 2005, present findings). The distribution of molecules involved in stereocilia function and hair cell transduction was shown to differ between cochlear and other hair cell types (Corey et al. 2004). Therefore, Pcdh15 may not be essential for the structural development of vestibular hair cells. Although Pcdh15 may be essential for the structural development of cochlear hair cells, it may also play a more general role in the transduction process in both cochlear and vestibular organs. More study is needed to determine the role of Pcdh15 in vestibular hair cell function.

Using antibodies to the cytoplasmic domain of Pcdh15, it has been shown that the protein is expressed in the cochlear neuroepithelium and spiral ganglion in mice at P8 (Alagramam et al. 2001a) and in the embryonic ear (Murcia and Woychik 2001). Subsequently, using different antibodies to the cytoplasmic domain of Pcdh15, Ahmed et al. (2003) reported that Pcdh15 is expressed in the inner ear stereocilia from early development into adulthood in mice. They also reported labeling of Pcdh15 in the adult cochlear hair cell cuticular plate.
In previous studies, based on stereocilia morphology in Pcdh15 mutants, it was proposed that Pcdh15 plays a role in the morphogenesis of the stereocilia, specifically, in the organization of the stereocilia bundle on the apical surface of hair cells (Alagramam et al. 2001b; Raphael et al. 2001). A more recent report from Ahmed et al. (2003) suggests that Pcdh 15 could serve as a component of the lateral link protein. We did not observe lateral link damage in our specimens. However, we did not use methods specifically designed for lateral link preservation and observation. Therefore, direct evidence that Pcdh15 is a component of the link system will require further investigation, perhaps by direct observation of changes in the lateral links in Pcdh15 mutants by TEM and/or immunogold labeling with antibodies to the Pcdh15 extracellular domain.

Based on data from the studies cited above, it is clear that Pcdh15 is present early in hair cell development and is localized so as to suggest a role in the formation and organization of the stereocilia on cochlear hair cells. This is consistent with the present study's findings, which show that different mutations of Pcdh15 alter not only stereocilia development but also formation of the cuticular plate and do so with differing degrees of severity that depends on the nature and extent of gene mutation.

\section{ACKNOWLEDGMENTS}

This research was supported by The NIDCD Grant DC05385-01 (KNA). The authors would like to thank Mr. Kunnathu Paulose for assistance in processing tissues. We would also like to thank Dr. Chris Gilpin, Mr. George Lawton, and Mr. Tom Januszewski (UT Southwestern Medical Center, Molecular and Cellular Imaging Facility) for their technical assistance. We would like to thank Jesse Washington III at Case Western Reserve University for technical assistance.

\section{REFERENCES}

Ahmed ZM, Riazuddin S, Ahmad J, Bernstein SL, Guo Y, Sabar MF, Sieving P, Riazuddin S, Griffith AJ, Friedman TB, Belyantseva IA, WILCOX ER. PCDH15 is expressed in the neurosensory epithelium of the eye and ear and mutant alleles are responsible for both USH1F and DFNB23. Hum. Mol. Genet. 12:3215-3223, 2003.

Alagramam KN, Kwon HY, Cacheiro NLA, Stubbs L, Wright CG, ERWAY LC, WOYCHIK RP. A new mouse insertional mutation that causes sensorineural deafness and vestibular defects. Genetics 152:1691-1699, 1999.

Alagramam KN, Zahorsky-Reeves J, Wright CG, Pawlowski KS, Erway LC, STubbs L, Woychiк RP. Neuroepithelial defects of the inner ear in a new allele of the mouse mutation Ames waltzer. Hear. Res. 148:181-191, 2000. 
Alagramam KN, Murcia Cl, Kwon HY, Pawlowski KS, Wright CG, Woychik RP. The mouse Ames waltzer hearing-loss mutant is caused by mutation of Pcdh15, a novel protocadherin gene. Nat. Genet. 27:99-102, 2001a.

Alagramam KN, Yuan HJ, Kuehn MH, Murcia CL, Wayne S, Srisailpathy CRS, Lowry RB, Knaus R, Van Laer L, Bernier FP, Schwartz S, Lee C, Morton CC, Mullins RF, Ramesh A, Van Camp G, Hagemen GS, Woychik RP, Smith RJH. Mutations in the novel protocadherin PCDH15 cause Usher syndrome type 1F. Hum. Mol. Genet. 10:1709-1718, 2001b.

Alagramam KN, Stahl JS, Jones SM, Pawlowski KS, Wright CG. Characterization of vestibular dysfunction in the mouse model for usher syndrome type 1F. J. Assoc. Res. Otolaryngol. 6(2):106-118, 2005.

Ben Yosef T, Belyantseva IA, Saunders TL, Hughes ED, Kawamoto K, Van Itallie CM, Beyer LA, Halsey K, Gardner DJ, Wilcox ER, Rasmussen J, Anderson JM, Dolan DF, Forge A, Raphael Y, Camper SA, Friedman TB. Claudin 14 knockout mice, a model for autosomal recessive deafness DFNB29, are deaf due to cochlear hair cell degeneration. Hum. Mol. Genet. 12:2049-2061, 2003.
Corey DP, Garcia-Anoveros J, Holt JR, Kwan KY, Lin Sy, Vollrath MA, Amalfitano A, Cheung ElM, Derfler BH, Duggan A, Geleoc GSG, Gray PA, Hoffman MP, Rehm HL, Tamasauskas D, Zhang DS. TRPA1 is a candidate for the mechanosensitive transduction channel of vertebrate hair cells. Nature 432:723-730, 2004.

Francis HW, Ryugo DK, Gorelikow MJ, Prosen CA, May BJ. The functional age of hearing loss in a mouse model of presbycusis. II. Neuroanatomical correlates. Hear. Res. 183:29-36, 2003.

Ichimiya I, Suzuki M, Mogi G. Age-related changes in the murine cochlear lateral wall. Hear. Res. 139:116-122, 2000.

Murcia CL, Woychik RP. Expression of Pcdh15 in the inner ear, nervous system and various epithelia of the developing embryo. Mech. Dev. 105:163-166, 2001.

Raphael Y, Kobayashi KN, Dootz GA, Beyer LA, Dolan DF, Burmeister M. Severe vestibular and auditory impairment in three alleles of Ames waltzer ( $a v)$ mice. Hear. Res. 151:237-249, 2001.

SuZUKI ST. Recent progress in protocadherin research. Exp. Cell Res. 261:13-18, 2000. 